\title{
Caracterização da dor lombar em gestantes atendidas no Hospital Universitário de Brasília
}

\author{
Priscilla Fernandes Sant' ana* \\ Sabrina Sousa Freire \\ Aline Teixeira Alves** \\ Denise Ribeiro Rabelo da Silva ${ }^{* * *}$
}

\begin{abstract}
RESUMO - O objetivo deste artigo é caracterizar a dor lombar em gestantes atendidas no Hospital Universitário de Brasília (HUB), utilizando a versão brasileira do Questionário McGill de Dor. Trata-se de um estudo de caráter transversal descritivo, no qual foram selecionadas quarenta gestantes, assistidas pelo programa de pré-natal de baixo risco do HUB, entre setembro e outubro de 2006. Analisando a intensidade da dor presente, $47,50 \%$ sentiam dor fraca; $32,50 \%$ moderada; $15 \%$ forte; nenhuma violenta e $5 \%$ insuportável. As médias do número de palavras escolhidas e indice de classificação da dor total foram 14,15 ( $\mathrm{DP} \pm 2,93$ ) e 30,77 ( $\mathrm{DP} \pm 7,16$ ), respectivamente. Concluiu-se que a lombalgia, na maioria das gestantes, é presente.
\end{abstract}

Palavras-chave: Gestantes. Lombalgia. Questionário McGill de Dor.

\section{Characterization of the low back pain in pregnant women treated at the Brasília Universitary Hospital}

\begin{abstract}
The objective of this article is to characterize the low back pain in pregnant women treated at the Brasília Universitary Hospital (HUB), using the Brazilian Version of the McGill Pain Questionnaire. It is a descriptive transversal study in which forty pregnant women were selected among those who were being assisted by the low risk prenatal program at HUB, between September and October, 2006. Analyzing the present pain intensity, $47.5 \%$ felt slight pain; $32.5 \%$ moderate; $15 \%$ strong; $0 \%$ violent and 5\% insuportable. The averages of the number of words chosen and the total pain rating index were $14.15(\mathrm{SD} \pm 2.93)$ and $30.77(\mathrm{SD} \pm 7.16)$, respectively. It can be concluded that the low back pain in the majority of the interviewed pregnant women is present.
\end{abstract}

Keywords: Pregnant woman. Low back pain. McGill Pain Questionnaire.

\footnotetext{
Graduadas em Fisioterapia - UniCEUB

** Especialista em Saúde da Mulher e Professora do Curso de Fisioterapia - UniCEUB

*** Especialista em Ortopedia e Traumatologia e Professora do Curso de Fisioterapia - UniCEUB deniserabelo@gmail.com
} 


\section{Introdução}

A gravidez é um episódio fisiológico com intensas alterações musculoesqueléticas, físicas e emocionais na vida da mulher, cujo organismo se adapta às modificações gerais e locais que ocorrem ao longo de sua evolução. Segundo Watanabe et al. (2000), uma gestação completa abrange cerca de 280 dias após o início do último período menstrual ou, aproximadamente, 40 semanas divididas em três períodos de três meses. O primeiro trimestre compreende da $1^{\mathrm{a}}$ a $13^{\mathrm{a}}$ semana, o segundo inclui da $14^{\mathrm{a}}$ a $26^{\mathrm{a}}$ semana, e o terceiro trimestre vai da $27^{\mathrm{a}}$ até a $38^{\mathrm{a}}$, a $39^{\mathrm{a}}$ ou a $40^{\mathrm{a}}$ semana.

As alterações que mais acarretam dor na gestante são as musculoesqueléticas, entre elas a cervicalgia, a dorsalgia, a lombalgia e a dor sacroilíaca (MARTINS; SILVA, 2005). Entende-se por lombalgia toda condição de dor, dolorimento ou rigidez que se localize na região inferior do dorso, situada entre o último arco costal e a prega glútea (CECIN et al., 1996). A etiologia de dor lombar específica na gravidez é mal definida, e sua origem é desconhecida. A lombalgia na gravidez inclui dor na área da coluna lombar, na pelve posterior e na sínfise púbica, podendo ser referida em região de perna, pé, região sacral e ou glútea. Esta condição pode ocorrer simultaneamente ou em diferentes momentos na mesma gestante, durante o período gestacional e após o parto. É importante identificar o tipo de dor para a melhor escolha do tratamento.

Tal definição não mostra um diagnóstico ou causa (SOUSA et al., 2003; ÖSTGAARD, 1996). Autores relatam que, aproximadamente, 50\% das mulheres experimentam algum tipo de dor durante a gravidez, geralmente em região lombar (CARLSON et al., 2003; ÖSTGAARD, 1996; STUGE et al., 2003). Martins e Silva (2005) identificaram que cerca de $80 \%$ das gestantes sentem dor na coluna vertebral, em algum período da gestação, sendo os locais de maior prevalência a região lombar e ou a sacroilíaca. Nove a quinze por cento dos casos são severos (STUGE et al., 2003).

Ronald Melzack, em 1975, apresentou um instrumento multidimensional de avaliação sistemática das queixas de dor que permite a qualificação e a quantificação: o Questionário McGill de Dor (The McGill Pain Questionnaire - (MPQ), que caracteriza três dimensões fundamentais da dor: sensorial-discriminativa, afetiva-motivacional e avaliativa-cognitiva (CASTRO, 1999). 
Existem poucos estudos que, além de demonstrarem a proporção de dor lombar, acrescentam características a esta dor (MARTINS; SILVA, 2005; CECIN et al., 1992). Não foram encontrados outros estudos que caracterizem a dor lombar em gestantes através da aplicação do Questionário McGill de Dor.

O objetivo deste trabalho é caracterizar a dor lombar em gestantes atendidas no Hospital Universitário de Brasília (HUB), utilizando a versão brasileira do Questionário McGill de Dor e dados, tais como: variação de peso, queixa de dor lombar prévia, idade, primiparidade, multiparidade e idade gestacional em semanas completas.

\section{Metodologia}

Trata-se de um estudo de caráter transversal descritivo, para o qual foram selecionadas, em amostra por conveniência, quarenta gestantes, sem limite de idade, assistidas pelo programa de pré-natal de baixo risco do Hospital Universitário de Brasília (HUB), no período matutino, às quartas e às quintas-feiras, entre $27 \mathrm{de}$ setembro e 18 de outubro de 2006. Previamente, foi entregue uma carta à chefe da Ginecologia e Obstetrícia do HUB, com pedido de liberação para que as grávidas pudessem ser entrevistadas pelas autoras desse estudo.

O critério de inclusão limitou-se à detecção da dor lombar nas gestantes, no momento da aplicação do questionário. Foram excluídas do estudo gestantes que não relataram presença de dor lombar no momento da aplicação do questionário, que tinham tomado analgésico nas últimas quatro horas e ou que faziam algum tratamento para a lombalgia, já que isto poderia mascarar o quadro de dor a ser avaliado.

Para a coleta de dados, foi aplicada a versão brasileira do Questionário McGill de Dor (QDM), traduzida em novembro de 1999, por Carlos Eduardo dos Santos Castro e dividida em quatro partes. A primeira é um esboço do corpo humano em que a paciente localiza a dor e a identifica como superficial ou profunda.

A segunda parte é formada por 68 palavras, divididas nas três dimensões principais da dor, e uma categoria mista de palavras, divididas em vinte subclasses contendo de duas a cinco palavras, para descrever diferentes aspectos da dor, com variação em intensidade de modo progressivo. Entre as 68 palavras, a paciente escolhe as que mais caracterizam a dor. Em cada subclasse, só pode ser mobilizada, no máximo, 
uma expressão, portanto, se, em alguma delas, a expressão não se aplicar àquela sentida pela paciente, não deverá ser marcada. Éssa escolha permite qualificar e quantificar a experiência dolorosa.

A terceira parte consiste em três conjuntos de palavras que descrevem a variação em intensidade que a percepção da dor pode sofrer em certo período de tempo. A última parte é formada por cinco palavras que avaliam a Intensidade da Dor Presente (PPI), ou seja, aquela que a paciente sente no momento da aplicação do questionário (CASTRO, 1999).

Foram coletados dados relacionados à idade, ao peso na primeira consulta, ao peso atual, à idade gestacional em semanas, à atividade laboral, ao número de gestações e à queixa de dor lombar prévia, que, em caso afirmativo, as gestantes caracterizaram-na como fraca, moderada, forte, violenta ou insuportável. Não houve limite de tempo para aplicação do questionário.

As voluntárias foram abordadas em quatro palestras para gestantes, oferecidas pelo HUB, todas as quartas-feiras, e, em apenas uma quinta-feira, pelo projeto de extensão de odontologia para gestantes. Todas receberam orientações quanto ao objetivo deste trabalho, e as que aceitaram participar voluntariamente assinaram um termo de consentimento livre e esclarecido, explicando o total sigilo dos dados fornecidos, a não-obrigatoriedade e a ausência de pagamento pela participação na pesquisa. Em caso de algum desconforto ou interferência na gravidez ou no feto, elas teriam o livre arbítrio para interromper o estudo. A aplicação do questionário foi realizada, individualmente, pelas pesquisadoras responsáveis por este estudo.

Os dados foram avaliados em outubro de 2006, por meio dos cálculos da média, do desvio padrão, da mediana e da frequiência das variáveis. O projeto foi aprovado pelo Comitê de Ética em Pesquisa do UniCEUB, no dia dezenove de setembro de 2006, protocolo 90/06, conforme resolução CNS 196/96.

\section{Resultados}

Entre as 40 gestantes entrevistadas, 15 (37,50\%) eram primíparas, e 25 (62,50\%), multíparas, independentemente de terem levado a gestação até o fim. Observou-se como média de idade 27,37 anos (DP $\pm 4,62$ ), com variação de 18 a 36 
anos. Para facilitar a análise dos dados, as idades encontradas na amostra foram divididas em 4 grupos com intervalos de 5 anos. Com isso, encontrou-se, $9(22,5 \%)$ gestantes com idade entre dezoito e vinte e dois anos, 11 (27,5\%) entre 23 e 27, 10 (25\%) com idade de 28 a 32 e $10(25 \%)$ de 33 a 37 anos. Em relação à idade gestacional, em semanas completas, a média foi de 19,77 semanas (DP $\pm 8,36$ ), sendo que $17(42,5 \%)$ estavam no $1^{\circ}$ trimestre, $10(25 \%)$, no $2^{\circ}$ trimestre, e $13(32,5 \%)$, no último trimestre.

Obtiveram ganho de peso 26 gestantes; em 8, não houve alteração, e 6 emagreceram até a data da coleta de dados. A mediana de variação de peso, expressa na Figura 1, em forma de Box Plot, foi, aproximadamente, 3,46 kg, sendo que uma gestante, na $10^{\mathrm{a}}$ semana gestacional, perdeu $3 \mathrm{~kg}$, e outra, na $33^{\circ}$ semana, ganhou $19 \mathrm{~kg}$.

Figura 1 - Variação de peso gestacional

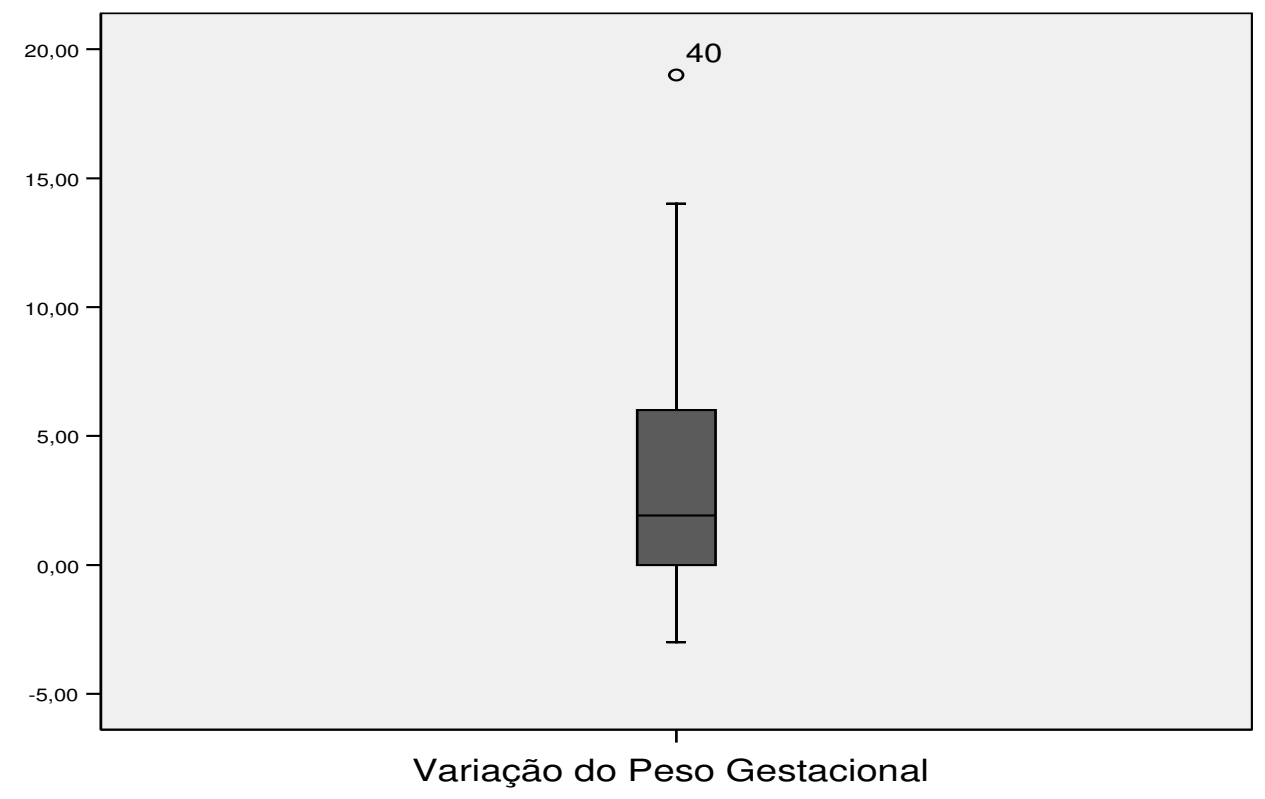

Relataram sentir dor lombar antes da gestação 20 (50\%) gestantes, sendo que 5 (25\%) delas classificaram a dor como fraca, 8 (40\%), como moderada, 6 (30\%), forte, nenhuma, violenta, e uma (5\%) classificou a dor lombar prévia como insuportável.

Na primeira parte do Questionário McGill de Dor, as gestantes foram instruídas a apontar no desenho o local da dor lombar. Duas delas disseram sentir, além da lombalgia, dor na região torácica. 
Analisando a Intensidade da Dor Presente (PPI), 19 (47,50\%) gestantes sentiam, no momento da aplicação do questionário, dor fraca; 13 (32,5\%), moderada; 6 (15\%), forte; nenhuma relatou dor violenta; apenas 2 (5\%), insuportável. Em relação ao Número de Palavras Escolhidas (NWC), as gestantes escolheram, aproximadamente, $70 \%$ do total de 20 palavras possíveis para descrição da dor, a média de 14,15 (DP \pm 2,93) palavras. Elas usaram, no mínimo, 8 e, no máximo, 19 expressões para caracterizar a dor.

De acordo com o Índice de Classificação da Dor (PRI), as médias encontradas foram: Sensorial 17,47 (DP $\pm 3,77)$, Afetiva 6,62 (DP $\pm 3,01)$, Avaliação Subjetiva 2,27 $(\mathrm{DP} \pm 0,72)$, Mistas 4,32 (DP $\pm 1,60)$ e o valor total de 30,77 (DP $\pm 7,16)$. A freqüência de cada subclasse do Índice de Classificação da Dor (PRI) está expressa na tabela 1.

Ao analisar a categoria Sensorial, as expressões das subclasses mais marcantes foram: Temporal, na qual a expressão "latejante" foi mobilizada por 19 (47,5\%) gestantes; Compressão, sendo "dor em câimbra/cólica" em 20 (50\%) pacientes; Tração, com maior concentração na expressão “que repuxa”, escolhida por 19 (47,5\%) grávidas; Calor, em relato como "dor que esquenta" por metade (50\%) das gestantes. Nas subclasses Incisão e Surdez, nenhuma expressão foi escolhida, totalizando 24 (60\%) e $18(45 \%)$ grávidas, respectivamente.

Entre as 5 subclasses da categoria Afetiva, em 3 delas as gestantes optaram por nenhuma palavra: Autonômica, em 23 (57,5\%) gestantes; Medo, em 24 (60\%); Punição, em 20 (50\%) pacientes da amostra. Relataram "dor incômoda" na Avaliação Subjetiva $25(62,5 \%)$ gestantes. Na categoria Mistas Sensoriais, a expressão "que cresce e diminui" foi mobilizada por 21 (52,5\%) mulheres; na categoria Mistas de Frio, 22 (55\%) mulheres não escolheram qualquer palavra; em Mistas Emocionais, 22 (55\%) gestantes optaram por "dor que deixa tensa".

\section{Discussão}

Estudos demonstram que a etiologia da dor lombar em mulheres grávidas é multifatorial. Entre as causas mais citadas, estão as hormonais, as biomecânicas e as vasculares (FERREIRA; NAKANO, 1999, 2000; SOUSA et al., 2003; CARLSON et al., 2003). 
Tabela 1 - Análise do Índice de Classificação da Dor (PRI)

\begin{tabular}{|c|c|}
\hline DESCRIÇÃO DA DOR & FREQÜÊNCIA (\%) \\
\hline \multicolumn{2}{|l|}{ SENSORIAL } \\
\hline Temporal & 95,00 \\
\hline Espacial & 72,50 \\
\hline Pressão - Ponto & 80,00 \\
\hline Incisão & 40,00 \\
\hline Compressão & 82,50 \\
\hline Tração & 77,50 \\
\hline Calor & 70,00 \\
\hline Vivacidade & 70,00 \\
\hline Surdez & 55,00 \\
\hline Geral & 97,50 \\
\hline \multicolumn{2}{|l|}{ AFETIVA } \\
\hline Cansaço & 87,50 \\
\hline Autonômica & 42,50 \\
\hline Medo & 40,00 \\
\hline Punição & 50,00 \\
\hline Desprazer & 95,00 \\
\hline AVALIAÇÃO SUBJETIVA & 85,00 \\
\hline \multicolumn{2}{|l|}{ MISTAS } \\
\hline Dor/Movimento & 60,00 \\
\hline Sensoriais & 77,50 \\
\hline De Frio & 45,00 \\
\hline Emocionais & 80,00 \\
\hline
\end{tabular}

Hormonais: secreção de relaxina pelo corpo lúteo, com maior intensidade no $1^{\circ}$ trimestre, levando à frouxidão ligamentar, o que justifica o aparecimento da dor precocemente. 
Biomecânicas: aumento de peso e volume abdominal, deslocamento anterior do centro de gravidade, pressão direta do feto, útero gravídico sobre as raízes nervosas da coluna lombossacra e tensão dos músculos espinhais antigravitacionais.

Vasculares: diminuição do fluxo sanguíneo medular, acarretando dor, principalmente no último trimestre.

Estudos, como os de Berg et al. (1988), Mantle et al. (1977), Mogren e Pohjanen (2005) e Melzack e Belanger (1989), evidenciaram que mulheres com lombalgia antes da gestação têm maior chance de desenvolvê-la na gravidez. Assim como Galão et al. (1995), este estudo não encontrou relação entre a dor prévia e o aparecimento ou o agravamento durante o período gestacional, pois foi encontrada a mesma proporção entre queixa e ausência de dor lombar antes da gravidez; além disso, não foi realizada estatística suficiente para analisar a possível correlação. Sihvonen et al. (1998) mostraram que a dor prévia está relacionada com a lombalgia na gestação, porém não há relação com a intensidade.

Encontramos maior frequiência de lombalgia em mulheres multíparas. Porém, é preciso frisar que a população estudada é pequena e apresenta amostra selecionada por conveniência. Segundo Östgaard (1996), Bouhassoun et al. (1998) e Galão et al. (1995), o número de gestações também constituiria fator de risco para o aparecimento da lombalgia gestacional, o que diverge de outros estudos, como os de Fast et al. (1987) e Melzack e Belanger (1989) que não encontraram a mesma correlação. No entanto, isso não significa que as primíparas tenham menor chance de desenvolverem a dor lombar (MELHADO et al., 2004). Para melhor avaliação da relação entre número de gestações e dor lombar, seria necessária a realização de novos estudos.

Alguns trabalhos mostram que, quanto menor a idade, maior a chance de ter dor lombar na gestação (KRISTIANSSON et al., 1996; MARTINS; SILVA, 2005; ÖSTGAARD et al., 1991; WANG et al., 2004). Enquanto outros (MANTLE et al., 1977) observaram que, com o aumento da idade, há maior prevalência de lombalgia. Orvieto et al. (1994) não encontraram correlação entre a idade materna e a lombalgia gestacional. No presente trabalho, encontrou-se maior concentração de gestantes, no total de 11 entre 23 e 27 anos.

Quando se relacionou lombalgia à idade gestacional, Martins e Silva (2005) verificaram que a dor foi mais prevalente no início da gestação e obtiveram o mesmo 
resultado observado no presente estudo, porém, para esse dado, não foi realizada razão de prevalência. Um estudo em que se utilizou a Escala Visual Analógica (VAS) para avaliação da intensidade da lombalgia na gestação, constatou que esta foi maior no $1^{\circ} \mathrm{e}$ no $3^{\circ}$ trimestre gestacional (SIHVONEN et al., 1998). Assis et al. (2004) observaram que a maior prevalência está no segundo trimestre, e Kristiansson et al. (1996) mostraram que a incidência aumenta a partir da $24^{\mathrm{a}}$ semana de gestação. Segundo Orvieto et al. (1994), a frequiência foi maior nas gestantes que estavam no último mês. Para Carvalho (2001), não há relação significativa entre os trimestres gestacionais.

Com relação ao ganho de peso durante a gestação, Ostgaard et al. (1994) evidenciaram que, com o aumento do peso, há um maior risco de desenvolver lombalgia gestacional. Foi observado neste estudo que mais da metade das gestantes obteve ganho de peso, todavia, não se pode afirmar que mulheres que aumentam o peso durante a gravidez irão, necessariamente, apresentar dor lombar, pois não houve uma análise estatística para esse dado. No entanto, nos estudos de Bouhassoun et al. (1998) e Heckman et al. (1994), não foi observada relação entre ganho de peso e ocorrência de dor lombar.

Não foi encontrado, na literatura, outro estudo de avaliação da dor lombar em gestantes, por meio do Questionário McGill de Dor, o que dificultou a comparação dos dados específicos deste trabalho com os dados obtidos em outros estudos de avaliação da dor. Grande parte da literatura disponível sobre este assunto realiza a avaliação da dor unidirecional, baseada apenas na intensidade da dor, como a Escala Visual Analógica, cujo exemplo, foi o estudo realizado por Sihvonen et al., (1998). Atualmente, o Questionário McGill de Dor é considerado um dos melhores métodos para caracterização da dor, pois permite a quantificação e a qualificação da dor, e por por isso foi o instrumento escolhido para a realização desta pesquisa.

\section{Conclusões}

Com base na análise da amostra, conclui-se que as multíparas, as gestantes entre 23 e 27 anos e as que se encontraram no $1^{\circ}$ trimestre de gestação apresentaram maior freqüência de dor lombar. Porém, esses dados só podem ser considerados válidos para a amostra estudada. 
Analisando o Questionário McGill de Dor, observa-se que a maioria das gestantes sentiu dor fraca. Na avaliação do Número de Palavras Escolhidas (NWC), aproximadamente, $70 \%$ das palavras possíveis foram escolhidas.

No Índice de Classificação da Dor (PRI), as subclasses que obtiveram maior frequiência foram: Temporal, Espacial, Pressão - Ponto, Compressão, Tração, Calor, Vivacidade, Surdez, Geral, Cansaço, Punição, Desprazer, Avaliação Subjetiva, Dor/ Movimento, Sensoriais, Emocionais. O grande número de escolha, 16 em 20 subclasses possíveis, parece indicar que, neste estudo, a dor lombar é um fator de grande desconforto durante a gravidez. As mulheres escolheram as expressões, com maior freqüência, em cinco das vinte subclasses: Sensorial Compressão, Sensorial Calor, Avaliação Subjetiva, Mistas Sensoriais e Mistas Emocionais. Pode-se dizer que a lombalgia da maioria das gestantes avaliadas neste estudo "que cresce e diminui, ocorre "em câimbra/cólica", "esquenta", "incomoda" e "deixa tensa", pois foram as expressões que apresentaram maior freqüência.

Os objetivos propostos por este trabalho foram cumpridos. No entanto, a do estudo faz-se necessária por ser oportuna a análise estatística que correlacione os dados obtidos na aplicação do Questionário McGill de Dor com os aspectos apresentados. Além disso, também é necessária a realização de outros estudos com amostra mais significativa, utilizando o mesmo instrumento do presente trabalho, com o propósito de aperfeiçoar a caracterização da dor lombar entre gestantes.

\section{Referências}

ASSIS, R.G.; TIBÚRCIO, R.E.S. Prevalência e características da lombalgia na gestação: um estudo entre gestantes assistidas no programa de pré-natal da maternidade dona Íris em Goiânia. Disponível em: <http://agata.ucg.br/formularios/neps/monografia/monografia_13>. Acesso em: 14 mai. 2004.

BERG, G. et al. Low back pain during pregnancy. Obstetrics and Gynecology, v. 71, n. 1, p. 71-75, 1988.

BOUHASSOUN N.; CATANZARITI J.F.; THÉVENON A. Lombalgies et grossesse. Revue du Rhumatisme, v. 65, p. 59S-62S, 1998. 
CARLSON, H.L. et al. Understanding and managing the back pain of pregnancy. Sports Medicine and Orthopedics, v. 3, p. 65-71, 2003.

CARVALHO, Y.B.R.; CAROMANO, F.A. Alterações morfofisiológicas relacionadas com lombalgia gestacional. Arquivos de Ciências da Saúde da Unipar, v. 5, n. 3, p. 267-272, 2001.

CASTRO, C.E.S. A Formulação lingüística da dor: versão brasileira do questionário McGill de dor. 1999. Dissertação (Mestrado), Universidade Federal de São Carlos, São Carlos, 1999. 234p.

CECIN, H. A. Coluna cervical e lombar. In: MOREIRA, C.; CARVALHO, M. A. .P. Noções práticas de reumatologia. Belo Horizonte: Editora Health, 1996. p. 181-204.

CECIN, H. A. et al. Lombalgia e gravidez. Revista Brasileira de Reumatologia, v. 32, n. 2, p. 45-50, 1992.

FAST, A. et al. Low back pain in pregnancy. Spine, v. 12, n. 4, p. 368-371, 1987.

FERREIRA, C. H. J.; NAKANO, A. M. S. Lombalgia na gestação: uma revisão. Jornal Brasileiro de Medicina, v. 77, n. 1, p. 113-118, 1999.

FERREIRA, C. H. J.; NAKANO, A. M. S. Lombalgia na gestação: etiologia, fatores de risco e prevenção. Femina, v. 28, n. 8, p. 435-438, 2000.

GALÃO, A. O.; ZARDO, E. A.; PAUlA L. G. Lombalgia da gestação. Acta Médica, v. 1, p. 347-353, 1995.

HECKMAN, J. D.; SASSART, R. Current concepts review: musculoskeletal considerations in pregnancy. The Journal of Bone and Joint Surgery, v. 76, p. 17201730, 1994.

KRISTIANSSON, P.; SVÄRDSUDD, K.; VON SCHOULTZ, B. Back pain during pregnancy. Spine, v. 21, p. 702-709, 1996.

MANTLE, M. J.; GREENWOOD, R. M.; CURRY, H. L. F. Backache in pregnancy. Rheumatology and Rehabilitation., v. 16, p. 95-101,1977.

MARTINS R. F.; SILVA J. L. P. Prevalência de dores nas costas na gestação. Revista da Associação Médica Brasileira, v. 51, n. 3, p. 144-147, 2005.

MELHADO, S. J. C.; SOLER, Z. A. G. A Lombalgia na gravidez: análise entre gestantes no último trimestre da gestação. Femina, v. 32, n. 8, p. 647-652, 2004.

MELZACK, R.; BELANGER, E. Labour pain: correlations with menstrual pain and acute low back pain before and during pregnancy. Pain, v. 36, p. 209-225, 1989. 
MOGREN, I. M.; POHJANEN, A. I. Low back pain and pelvic pain during pregnancy: prevalence and risk factors. Spine, v. 30, n. 8, p. 983-991, 2005.

ORVIETO, R.; ACHIRON, A.; BEM-RAFAEL, Z.; GELERNTER, I.; \& ACHIRON, R. Low back pain of pregnancy. Acta Obstetricia Et Gynecologica Scandinavica., v. 73, p. 209-214, 1994.

ÖSTGAARD, H. C.; ANDERSSON, G. B. J.; KARLSSON, K. Prevalence of back pain in pregnancy. Spine, v. 16, n. 5, p. 549-552, 1991.

ÖSTGAARD, H. C.; ZETHERSTROM, G.; ROSS-HANSEN, E.; SVANBERG, G. Reduction of back and posterior pelvic pain in pregnancy. Spine, v. 19, n. 8, p. 894-900, 1994.

OSTGAARD, H. C. Assessment and treatment of low back pain in working pregnant women. Spine, v. 20, n. 1, p. 61-69, 1996.

SIHVONEN, T.; HUTTUNEN, M.; MAKKANEN, A.; AIRAKSINEN, O. Functional changes in back muscle activity correlate with pain intensity and prediction of low back pain during pregnancy. The Archives of Physical Medicine and Rehabilitation, v. 79, p. 1210-1212, 1998.

SOUSA, M. S.; ARAUJO, C. C.; CASTELLEN, M.; SPERANDIO,F. F. Afinal, toda gestante terá dor lombar? Femina, v. 31, n. 3, p. 273-277, 2003.

STUGE, B.; HILDE, G.; VOLLESTAD, N. Physical therapy for pregnancy-related low back and pelvic pain: a systematic review. Acta Obstetricia Et Gynecologica Scandinavica, v. 82, p. 983-990, 2003.

WANG, S. M.; DEZINNO, P.; MARANETS, I.; BERMAN, M. R.; CALDWELLANDREWS, A. A.; KAIN, Z. N. Low back pain during pregnancy: prevalence risk factors and outcomes. Obstetrics and Gynecology, v. 104, n. 1, p. 65-70, 2004.

WATANABE, E. K. Evolução cronológica do concepto: duração da prenhez. In: BUSSÂMARA, N. Obstétrica Básica. 2o ed., São Paulo: Sarvier, 2000. p.66-69. 